\title{
Light scattering described in the mode picture
}

\author{
W. Winkler, R. Schilling, K. Danzmann, J. Mizuno, A. Rüdiger, and K. A. Strain
}

\begin{abstract}
A system of orthonormal functions representing the eigenmodes of an optical resonator with perfectly spherical mirror surfaces has been described in the literature. In real experiments, however, the wave front of the passing beam will be deformed by surface irregularities or index inhomogeneities inside components traversed by the beam. We describe quantitatively the relative power transferred out of the fundamental mode into higher-order modes by these irregularities.

Key words: Wave-front deformations, light scattering, modes.
\end{abstract}

\section{Introduction}

The commonly used description of the scattering of light assumes an infinitely wide plane wave incident upon a nonperfect optical component. ${ }^{1}$ The imperfection may be a fluctuation in the index of refraction of a substrate traversed by the beam, or it may be a deviation of a surface from its ideal shape, such as a surface ripple on top of a sphere. Any deviation from wave-front propagation as determined by ideally shaped components may be called scattering. The scattering properties of a component are commonly described by the relative power of light scattered into a given solid angle. This is a function of the angle of incidence, the scattering angle (i.e., the angular deviation from the ideal path), and the polarization of the incident wave. We restrict ourselves here to the most important case of essentially normal incidence and small distortions. Then the polarization is not changed.

The well-known theory ${ }^{2}$ of scattering by an amplitude or phase grating relates the scattering angle, $\Theta$, to the spatial wavelength, $\Lambda$, of the irregularity by $\Theta=\lambda / \Lambda ; \lambda$ is, as usual, the wavelength of the light. Sometimes the total relative power deviating from the ideal path, the so-called total integrated scattering (TIS), is given. In the case of reflection at a

When this work was performed, the authors were with the Max-Planck-Institut für Quantenoptik, D-85748 Garching bei Munchen, Germany. K. Danzmann is now also with the Institut für Atom-und Molekülphysik, Universität Hannover, Appelstrasse 2, D-30167 Hannover, Germany; K. A. Strain is with the Department of Physics and Astronomy, University of Glasgow, Glasgow G12 8QQ, United Kingdom.

Received 16 December 1993; revised manuscript received 4 May 1994.

0003-6935/94/317547-04\$06.00/0.

(1) 1994 Optical Society of America. distorted surface, its magnitude is related to the root-mean-square deviation, $h_{\text {rms }}$, of the surface with respect to the ideal shape by

$$
\text { TIS }=\left(\frac{4 \pi h_{\mathrm{rms}}}{\lambda}\right)^{2} .
$$

For a beam of finite width this definition is somewhat problematic, as it is not clear up to what spatial wavelength the average has to be taken. One expects the upper limit of relevant spatial wavelengths to be roughly corresponding to the beam diameter. However, here factors of the order of 2 are fairly important, as the spectral density of the amplitude of surface deformations (as well as of fluctuations in refractive index) normally increases strongly toward longer spatial wavelengths. ${ }^{3}$

The description of the effect of a wave-front deformation by a single number such as the TIS or the scattering angle, above which the light is considered as being lost from the original mode, is a relatively poor one and might not be adequate for all applications. A more appropriate approach is to use the mode picture, in which a wave-front deformation is considered to cause coupling into other modes; the deformed beam is described as a superposition of the original (fundamental) mode and higher-order modes. In this way, for instance, it is possible to model a real cavity to a good approximation. In particular, it allows us to describe the suppression of scattering into higher modes, if these are not resonant. Thus scattering may be strongly reduced; this is an important issue, for instance, in interferometric gravitational wave detectors with recycling schemes implemented. ${ }^{4}$

In this paper we present a treatment of scattering in the framework of orthonormal optical modes; in a following paper the same approach will be applied to 
the effect of thermal lensing. For our present purpose a one-dimensional consideration is sufficient; the formalism for two dimensions can be found in a paper by Bayer-Helms. ${ }^{5}$

\section{Orthonormal Optical Modes}

A system of orthonormal functions representing the eigenmodes of an optical cavity was originally found by Fox and $\mathrm{Li}^{6}$ and by Boyd and Kogelnik ${ }^{7}$ and is now contained in textbooks. ${ }^{2}$ For simplicity we omit in the following the curvature of the wave front of the undistorted modes. In one dimension, normalized appropriately, these modes can then be written as

$$
\psi_{n}(u)=\exp \left(-u^{2} / 2\right) \frac{H_{n}(u)}{\left(n ! 2^{n} \sqrt{\pi}\right)^{1 / 2}} .
$$

As usual, the beam radius (measured between beam axis and the $1 / e^{2}$ point of the intensity) is called $w$; the transverse coordinate, $u$, used here is normalized to $w / \sqrt{2}$ and thus is dimensionless. The $H_{n}$ are the Hermitian polynomials defined by

$$
H_{n}(u)=(-1)^{n} \exp \left(u^{2}\right) \frac{\mathrm{d}^{n}}{\mathrm{~d} u^{n}} \exp \left(-u^{2}\right) .
$$

The lowest-order Hermitian polynomials are

$$
\begin{aligned}
& H_{0}(u)=1 ; \quad H_{1}(u)=2 u ; \quad H_{2}(u)=4 u^{2}-2 ; \\
& H_{3}(u)=8 u^{3}-12 u ; \quad \cdots .
\end{aligned}
$$

In this form the eigenfunctions $\psi_{n}(u)$ are orthonormal:

$$
\int_{-\infty}^{+\infty} \psi_{m}(u) \psi_{n}(u) \mathrm{d} u=\delta_{m n},
$$

with $\delta_{m n}$ being the Kronecker symbol.

The fundamental mode is

$$
\psi_{0}(u)=\frac{\exp \left(-u^{2} / 2\right)}{\sqrt[4]{\pi}} .
$$

The size of the higher modes increases with the mode order, $n$; for large $n$, radius $w_{n}$ is related to radius $w_{0}$ of the fundamental mode by

$$
w_{n} \approx \sqrt{n} w_{0} \text {. }
$$

For $n \geq 1$, the amplitude of the beam shows a sinusoidal behavior when scanning across the wave front; the $n$th mode has $n$ zero crossings, and therefore the spatial intensity distribution shows fringes. For large $n$, spatial wavelength $\Lambda_{n}$ of this amplitude variation across the wave front approaches

$$
\Lambda_{n} \approx \frac{4 w_{0}}{\sqrt{n}}
$$

All these modes change their size with distance, but not their shape.

\section{Coupling of Modes by Wave-Front Deformation}

In general, the deformation imposed by a real system onto the wave front of a passing beam is a complicated two-dimensional function of the transverse coordinates. The distortion may be expanded into a twodimensional Fourier series. The two dimensions can be assumed as being independent from each other and thus being factorized. It is therefore sufficient to describe the power transfer from one mode into another one as it is caused by a single one-dimensional Fourier component. This component is a spatial sine wave with a given wavelength, amplitude, and spatial phase. The effect of a real wave-front deformation is then obtained by integration over the whole (two-dimensional) spectrum.

In a practical case (with no preferred direction of the deformations), even a simplified procedure of averaging the spectra of parallel scan lines across the wave front gave results close to those obtained by a full two-dimensional decomposition of the field into higher-order modes. In this paper we therefore restrict ourselves to one dimension and a single spatial wavelength.

In many laser applications a system is illuminated by the fundamental mode. Let the beam be reflected either at a surface grating, realized by a harmonic deformation with spatial wavelength $\Lambda$, or pass through a substrate having a corresponding variation in index of refraction. It is convenient to define a normalized, spatial frequency $\Omega$ by

$$
\Omega=\sqrt{2} \pi w_{0} / \Lambda \text {. }
$$

The deformed wave front, $\psi(u)$, of the beam is then described by

$$
\psi(u)=\frac{\exp \left(-u^{2} / 2\right)}{\sqrt[4]{\pi}} \exp \left\{-i\left[\vartheta u+\phi_{0} \cos (\Omega u+\Phi)\right]\right\}
$$

where $\phi_{0}$ is the peak value of the phase modulation and $\Phi$ is its spatial phase. For $\Phi=0$ we have the case of a deformation symmetric to the beam axis; for $\Phi=\pi / 2$ the deformation is antisymmetric. For completeness, a tilt of the wave front was included, with the quantity, $\vartheta$, being related to the tilt, $\alpha$, by

$$
\vartheta=\frac{2 \pi}{\lambda} \frac{w_{0}}{\sqrt{2}} \alpha
$$

Tilt $\alpha$ of the wave front corresponds to tilt $\alpha / 2$ of a reflecting surface. In the case of reflection at a surface with a deformation amplitude of $h_{0}$, the peak 
value of the phase modulation, $\phi_{0}$, is given by

$$
\phi_{0}=4 \pi \frac{h_{0}}{\lambda},
$$

with $\lambda$ being the wavelength of the light.

\section{Generalized Total Integrated Scattering}

In order to get the (possibly complex) amplitude, $a_{0, d}$, of the fundamental mode contained in the distorted beam, one must fold the distorted field with the fundamental mode:

$$
\begin{aligned}
a_{0, d}= & \int_{-\infty}^{+\infty} \psi(u) \psi_{0}(u) \mathrm{d} u \\
= & \int_{-\infty}^{+\infty} \frac{\exp \left(-u^{2} / 2\right)}{\sqrt[4]{\pi}} \frac{\exp \left(-u^{2} / 2\right)}{\sqrt[4]{\pi}} \\
& \times \exp \left\{-i\left[\vartheta u+\phi_{0} \cos (\Omega u+\Phi)\right]\right\} \mathrm{d} u .
\end{aligned}
$$

For small tilts (such that $\vartheta u \ll 1$ across the beam) and for small values of deformations $\left(\phi_{0} \ll 1\right)$, we may expand the third exponential factor (with the imaginary argument) up to second order:

$$
\begin{aligned}
a_{0, d} & \approx \int_{-\infty}^{+\infty} \frac{\exp \left(-u^{2}\right)}{\sqrt{\pi}}\left[1-i \vartheta u-i \phi_{0} \cos (\Omega u+\Phi)\right. \\
& -\frac{1}{2} \vartheta^{2} u^{2}-\vartheta \phi_{0} u \cos (\Omega u+\Phi) \\
& \left.-\frac{1}{2} \phi_{0}^{2} \cos ^{2}(\Omega u+\Phi)\right] \mathrm{d} u .
\end{aligned}
$$

The relevant definite integrals can be found in mathematical handbooks ${ }^{8}$ to yield, after some straightforward algebra,

$$
\begin{aligned}
\frac{\Delta P_{0}}{P_{0}}= & \frac{\phi_{0}{ }^{2}}{2}\left[1-\exp \left(-\Omega^{2} / 2\right)\right]\left[1-\cos 2 \Phi \exp \left(-\Omega^{2} / 2\right)\right] \\
& +\frac{\vartheta^{2}}{2}-\vartheta \phi_{0} \sin \Phi \Omega \exp \left(-\Omega^{2} / 4\right)
\end{aligned}
$$

for the relative power lost from the fundamental mode.

If we are free to choose the tilt appropriately [see Eq. (10)], the minimum power loss from the fundamental mode is obtained with

$$
\vartheta_{\text {opt }}=\phi_{0} \sin \Phi \Omega \exp \left(-\Omega^{2} / 4\right) .
$$

The power loss from the fundamental mode is then

$$
\begin{aligned}
\frac{\Delta P_{0}}{P_{0}}= & \frac{\phi_{0}{ }^{2}}{2}\left\{\left[1-\exp \left(-\Omega^{2} / 2\right)\right]\left[1-\cos 2 \Phi \exp \left(-\Omega^{2} / 2\right)\right]\right. \\
& \left.-\frac{1}{2}(1-\cos 2 \Phi) \Omega^{2} \exp \left(-\Omega^{2} / 2\right)\right\} .
\end{aligned}
$$

The minimum amount of scattering is obtained when the beam is positioned such as to get a wavefront deformation symmetric to the beam axis, that is, with $\Phi=0$ :

$$
\frac{\Delta P_{0}}{P_{0}}=\frac{\phi_{0}{ }^{2}}{2}\left[1-\exp \left(-\Omega^{2} / 2\right)\right]^{2} .
$$

One can rewrite this relation for the case of reflection at an imperfect surface, using directly the quantities by which normally such surfaces are described [see Eqs. (8), (9), and (11)]:

$$
\frac{\Delta P_{0}}{P_{0}}=\left(\frac{4 \pi}{\lambda} h_{\mathrm{rms}}\right)^{2}\left\{1-\exp \left[-\left(\pi w_{0} / \Lambda\right)^{2}\right]\right\}^{2}
$$

This equation allows us to estimate, for beams of finite width, how to determine the value of $h_{\mathrm{rms}}$ that has to be used in calculating the TIS from Eq. (1) in case of arbitrary wave-front distortions. Particularly a corner wavelength, $\Lambda_{c}$, is given, up to which an average has to be taken to get the appropriate rms value of surface deformation relevant for scattering:

$$
\Lambda_{c}=\pi w_{0} .
$$

This is important for the definition of the specifications for optical components to be used in a system with low losses, particularly in the case of large beam diameters.

\section{Coupling into Higher Modes}

The relative amplitude of light scattered into the mode number, $n$, is similarly determined by folding the distorted mode with that particular higher mode:

$$
\begin{aligned}
a_{n}= & \int_{-\infty}^{+\infty} \psi(u) \psi_{n}(u) \mathrm{d} u \\
= & \int_{-\infty}^{+\infty} \frac{\exp \left(-u^{2} / 2\right) H_{n}(u)}{\left(n ! 2^{n} \sqrt{\pi}\right)^{1 / 2}} \frac{\exp \left(-u^{2} / 2\right)}{\sqrt[4]{\pi}} \\
& \times \exp \left\{-i\left[\vartheta u+\phi_{0} \cos (\Omega u+\Phi)\right]\right\} \mathrm{d} u .
\end{aligned}
$$

Again we assume $\vartheta_{u}$ and $\phi_{0}$ to be small. It is then sufficient to expand the exponential up to the first order. The relevant integrals can be found in tables ${ }^{8}$ to give, for $n \geq 1$,

$$
\begin{aligned}
a_{n}= & -\frac{i}{\sqrt{n ! 2^{n}}} \\
& \times\left[\delta_{1 n} \vartheta+\phi_{0} \cos \left(\Phi+n \frac{\pi}{2}\right) \Omega^{n} \exp \left(-\Omega^{2} / 4\right)\right] .
\end{aligned}
$$

As expected, a symmetric distortion excites only even modes, and an antisymmetric distortion excites only odd ones. In this approximation of small distortions, a tilt of the wave front couples only into the 


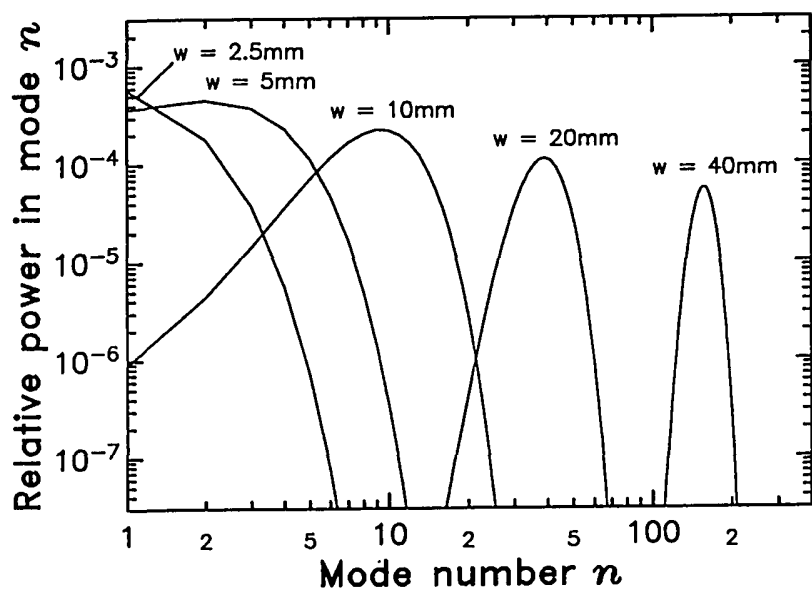

Fig. 1. Relative power transferred from the fundamental mode into modes of order $n$, with beam radius $w$ as a parameter for the different curves. A sinusoidal wave-front deformation with spatial wavelength $\Lambda=10 \mathrm{~mm}$ and an rms amplitude of $\lambda / 200$ is imposed onto the beam; in case of reflection at a deformed surface, this corresponds to an rms amplitude of the surface ripple of $\lambda / 400$. Note that, for a deformation symmetric to the beam, all odd modes disappear, for an antisymmetric deformation, all even modes disappear.

first mode; its power is

$$
P_{1}=\frac{\vartheta^{2}}{2}-\vartheta \phi_{0} \sin \Phi \exp \left(-\Omega^{2} / 2\right)
$$

Thus the first-order mode can always be made to disappear by the adjustment of the orientation of the beam axis relative to the substrate such as to fulfill Eq. (15).

The power in the modes $n \geq 2$ is given by

$$
P_{n}=\frac{1-(-1)^{n} \cos 2 \Phi}{2} \frac{\phi_{0}{ }^{2}}{n !}\left(\frac{\Omega^{2}}{2}\right)^{n} \exp \left(-\Omega^{2} / 2\right) \text {. }
$$

For a given spatial wavelength of wave-front deformation, Eq. (23) can be evaluated to determine the power distribution of the scattered light into the different higher modes. Figure 1 shows this distribution for a distortion with fixed spatial wavelength and a set of different beam diameters. The maximum power is deposited into the mode of order

$$
n_{\max }=\frac{\Omega^{2}}{2}=\left(\frac{\pi w_{0}}{\Lambda}\right)^{2} .
$$

For example, a wave-front deformation with a spatial wavelength equal to the beam radius deposits most of the scattered light into the modes around the order of 10 .

\section{Conclusion}

The mode picture provides a relatively simple framework for describing the scattering of laser beams caused by wave-front deformations, as long as the distortions are small enough. This is a condition that is satisfied in most of the relevant cases. It is the appropriate tool for calculating the losses of the fundamental mode as well as the power shifted into higher modes for a given wave-front deformation. The approach shown in this paper permits outlining the specifications for the manufacturers of highquality optics, in particular for cases in which the beam diameters are larger than a few millimeters.

\section{References}

1. P. Beckmann and A. Spizzichino, The Scattering of Electromagnetic Waves from Rough Surfaces (Macmillan, New York, 1963).

2. See, for example, A. E. Siegman, Lasers (University Science, Mill Valley, Calif., 1986).

3. W. Winkler, K. Danzmann, A. Rüdiger, and R. Schilling, "Optical problems in interferometric gravitational wave antennas," in The Sixth Marcel Grossmann Meeting, H. Sato and T. Nakamura, eds. (World Scientific, Singapore, 1992), pp. 176191.

4. B. Meers and K. A. Strain, "Wave-front distortion in laserinterferometric gravitational-wave detectors," Phys. Rev. D 43, 3117-3130 (1991).

5. F. Bayer-Helms, "Coupling coefficients of an incident wave and the modes of a spherical optical resonator in the case of mismatching and misalignment," Appl. Opt. 23, 1369-1380 (1984).

6. A. G. Fox and T. Li, "Resonant modes in a maser interferometer," Bell Syst. Tech. J. 40, 453-488 (1961).

7. G. D. Boyd and H. Kogelnik, "Generalized confocal resonator theory," Bell Syst. Tech. J. 41, 1347-1369 (1962).

8. See, for example, I. S. Gradshteyn and I. Ryzhik, Tables of Integrals, Series, and Products (Academic, London, 1980). 